\title{
«Diderot Studies», tome XXXV
}

\section{Eszter Kovács}

\section{OpenEdition}

\section{Journals}

Édition électronique

URL : http://journals.openedition.org/studifrancesi/15339

DOI : 10.4000/studifrancesi. 15339

ISSN : 2421-5856

\section{Éditeur}

Rosenberg \& Sellier

\section{Édition imprimée}

Date de publication : 1 décembre 2018

Pagination : 496-497

ISSN : 0039-2944

\section{Référence électronique}

Eszter Kovács, « «Diderot Studies», tome XXXV », Studi Francesi [En ligne], 186 (LXII | III) | 2018, mis en ligne le 01 janvier 2019, consulté le 06 janvier 2021. URL : http://journals.openedition.org/ studifrancesi/15339; DOI : https://doi.org/10.4000/studifrancesi.15339

Ce document a été généré automatiquement le 6 janvier 2021.

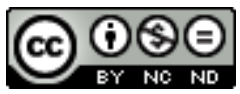

Studi Francesi è distribuita con Licenza Creative Commons Attribuzione - Non commerciale - Non opere derivate 4.0 Internazionale. 


\title{
«Diderot Studies», tome XXXV
}

\author{
Eszter Kovács
}

\section{RÉFÉRENCE}

«Diderot Studies», tome XXXV, Lire "Le Neveu de Rameau", éd. Thierry BELLEGUIC, Genève, Droz, 2015, $412 \mathrm{pp}$.

1 Le dernier volume des «Diderot Studies», daté de 2015, est entièrement consacré au Neveu de Rameau, sans doute pour préparer l'agrégation de lettres modernes de 2016-2017. Vu l'importance de cet ouvrage, nous avons jugé utile de le présenter aux lecteurs de «Studi Francesi», nonobstant le retard avec lequel nous avons été obligée de le faire. Le Neveu étant un des textes le plus souvent commentés de Diderot, il importait d'offrir des analyses ciblées ou novatrices, tout en évoquant certaines des critiques fondatrices qui les ont précédées. C'est ce que les différents auteurs qui ont collaboré à ce numéro de «Diderot Studies» ont fait, pour le plaisir et l'intérêt des lecteurs. Le volume se compose de dix-neuf articles traitant de la Satire seconde, ce qui peut paraître décourageant et difficile à lire à la suite même pour les spécialistes. On peut toutefois amplement puiser dans ce volume pour avoir une vue d'ensemble de nombreux aspects relatifs à cet ouvrage et à sa postérité.

2 Le premier article, The Nephew's time de Daniel BREWER (pp.3-23), démontre que la pensée se forme dans Le Neveu de Rameau en tant que parole et écoute. Lors de la rencontre entre Lui et Moi, le temps est présent en tant que kairos (le temps qui se transforme) et non pas en tant que chronos (le temps qui s'écoule). Anthony waLL, dans Diderot's chronophotographic writing in Rameau's Nephew (pp. 25-56), analyse la dimension visuelle de l'écriture: les images du dialogue sont en mouvement, elles s'enchaînent, ce qui revêt ce texte d'une dynamique particulière. Jean-Claude BouRDIN, dans Jean-François Rameau contre-philosophe (pp.59-96), revisite la lecture hégélienne du Neveu. Peut-on toujours lire ou relire Le Neveu comme une autocritique des Lumières? En effet, comme le confirme l'article, la bassesse de Lui l'emporte sur la prétendue hauteur morale de Moi et la reprise du topos reductio ad mortem minimise l'importance de la hiérarchie 
morale et sociale. Jean-Luc MARTINE, dans L'art et la matière: aperçus sur l'écriture du matérialisme (pp. 97-114), passe en revue certaines critiques connues, par exemple celles de Jean-Claude Bourdin et de Jean Starobinski, et applique les propositions d'olivier Bloch sur le matérialisme à la Satire seconde.

Comme le souligne Florence BOULERIE dans son article "Mes pensées, ce sont mes catins»: ambiguïtés et compromission de la philosophie dans "Le Neveu de Rameau" (pp. 115-136), le thème de l'avilissement, omniprésent dans Le Neveu, fascine toujours. Selon F. Boulerie, aucun des avis opposés dans le dialogue n'est vrai: il s'agit d'un texte sans vérité. JeanPierre CLÉRo montre dans Commerce, pesées et calculs dans "Le Neveu de Rameau" (pp.137-167), que ce dialogue représente la société comme un marché. Zeina НАКім, dans l'article Des bénéfices de la déraison (pp.169-177), reprend le problème de la subversion, un terme qui revient souvent à propos du Neveu de Rameau car la Satire seconde renverse les points de vue et les systèmes de valeurs. Dans l'interprétation de Zeina Hakim, Moi est un aliéné, un philosophe à sa manière. Paolo CASINI, dans son "Le Neveu de Rameau" ou les sables mouvants de la dialectique (pp. 179-195), dont l'enjeu est de revisiter "une tradition critique bicentenaire», nous offre un article méthodique et clair sur la réception à proprement parler philosophique du Neveu. Pascal Duc et Marian HOBSON, dans "Le Neveu de Rameau": Eighteenth-century music as a stepping stone to hegelian dialectic (pp.197-212), réexaminent, quant à eux, l'aspect musical du texte. Ils font mention d'un site (issu d'un projet) sur lequel dix-huit échantillons de musique auxquels le dialogue se réfère accompagnent le texte. En reconsidérant la réflexion musicale de Diderot, ils remarquent qu'il s'agit d'une «satire», l'opinion de Diderot reste donc insaisissable mais il incite certainement à un renouvellement musical.

Nombre de questions continuent de préoccuper lecteurs et critiques du Neveu de Rameau, notamment en ce qui concerne le rapport entre l'esthétique et l'éthique. Il n'est donc pas étonnant qu'une partie considérable du volume soit réservée à cet aspect. Baldine SAINT GIRONS, dans Y a-t-il un sublime dans le mal (pp. 213-233), réexamine cette question toujours déconcertante de la Satire seconde. Cornelia KLETTKE, dans l'article Le génie sublime et sa parodie (pp. 235-253), parcourt le concept de génie du $\mathrm{XVI}^{\mathrm{e}}$ siècle jusqu'aux écrits de Diderot. Alors que dans les ouvrages de jeunesse de Diderot, on voit clairement un culte du génie, cette réflexion devient plus tard plus complexe: on a tendance à voir dans la figure du Neveu un homme vilain mais original. Lucien NoUIs, dans La colère et la joie (pp. 255-292), montre à quel point Diderot mobilise les affections et les passions dans Le Neveu: Rameau, en faisant l'apologie des passions basses, démontre la proximité des passions et des vices. Jean-Alexandre PERRAS, dans Usages et réformes: "Le Neveu de Rameau" et les ambivalences de l'originalité (pp. 293-315), revisite le caractère et la caractérisation du Neveu: il est difforme, abject, mais «au moins original dans [son] avilissement», un original blâmable.

Michel Delon, dans "Le Neveu de Rameau" et la jolie femme (pp. 317-328), se concentre sur un détail, la jolie femme, symbole de l'inconstance, de la frivolité et du commerce mondain. Rameau en parle avec une sincérité cynique: selon lui, chacun se prostitue à sa manière dans une société corrompue. L'article de Kate E. TUNSTALL, "Le Neveu de Rameau", règne des magots et des pagodes (pp. 329-346), est consacré à certains détails présents dans le dialogue, à leur message et leur rôle. Le luxe est signe de corruption mais non pas pour le Neveu qui s'adonnerait volontiers à de tels plaisirs. L'article essaie de mettre en contexte la présence de ces détails - chinoiseries et objets de luxe - par rapport à la réflexion de Diderot dans d'autres textes, notamment les Salons et les écrits 
dédiés à Catherine II. L'article de Jeffrey LEICHMAN Quantum performance: intersubjectivity, uncertainity, and the "Neveu de Rameau" (pp. 347-366) peut étonner le lecteur: comment interpréter la référence au quantum physique? Il s'agit d'une rencontre-souvent difficile - entre théorie scientifique et théorie littéraire. Comment appliquer une théorie révolutionnaire dans l'histoire des sciences à une performance «littéraire»? L'auteur de l'article rapproche l'incertitude physique et morale (ou l'incertitude en physique et en morale) et constate que le dialogue expose cette instabilité.

Les trois derniers articles traitent de la réception du Neveu de Rameau. Dans Insights with Hindsight: Adapting "Le Neveu de Rameau" for the citizens theatre, Glasgow, 1998 (pp. 367-376), Phoebe voN HELD avance que mettre Le Neveu de Rameau en scène ne va pas de soi en dépit du caractère théâtral du texte. L'auteur de l'article justifie son propre essai de mise en scène. À quel point l'adaptation est-elle possible? Selon P. Von Held, il faut faire des choix et mettre en scène certains extraits (et certains aspects) de la Satire seconde et renoncer à la totalité pour éviter que la pièce soit un échec. Katalin BARTHAKovÁcs, dans "Le Neveu de Rameau" au miroir de sa réception hongroise (pp. 377-394), traite des temps forts de la réception de l'ouvrage dans la culture-cible et cherche à expliquer l'attention au Neveu de Rameau de temps en temps ressuscitée dans la vie intellectuelle hongroise. Dans L'expansion de la Mimésis: "Le Neveu de Rameau" et une légende biographique d'André Chénier (pp. 395-411), Serge ZENKINE évoque les derniers moments d'André Chénier, son geste et ses mots en se frappant le front («Mourir. J'avais quelque chose là»). Ce geste et ses paroles font penser à une scène dans Le Neveu. Le rapprochement est intéressant mais la relation n'est pas avérée, ce que l'auteur de l'article reconnaît de sa part.

7 Après avoir parcouru ces dix-neuf articles sur Le Neveu de Rameau, il est légitime de se poser la question: comment innover la critique sur cet ouvrage, tellement riche, tellement intrigant? Je voudrais attirer l'attention sur une seule des difficultés que cette tentative comporte: parfois ceux qui commentent Le Neveu veulent imiter ou égaler la verve de Diderot, sans toujours réussir. Les questionnements et les sujets des articles se recoupent assez souvent (subversion, folie, déraison, originalité, génie, le rire, la méta-éthique). J'ajoute qu'il aurait été utile d'insérer à la fin du volume un index de noms propres (pour retrouver facilement quels articles évoquent Goethe ou Hegel...). Philosophes et littéraires ne peuvent, en tout cas, que se réjouir de la parution de ce nouveau volume consacré à la Satire seconde de Diderot. 